\title{
CONVERGENCE RATE OF APPROXIMATE SOLUTIONS TO WEAKLY COUPLED NONLINEAR SYSTEMS
}

\author{
HAIM NESSYAHU ${ }^{1}$
}

\begin{abstract}
We study the convergence rate of approximate solutions to nonlinear hyperbolic systems which are weakly coupled through linear source terms. Such weakly coupled $2 \times 2$ systems appear, for example, in the context of resonant waves in gas dynamics equations.

This work is an extension of our previous scalar analysis. This analysis asserts that a One Sided Lipschitz Condition (OSLC, or Lip ${ }^{+}$-stability) together with $W^{-1,1}$-consistency imply convergence to the unique entropy solution. Moreover, it provides sharp convergence rate estimates, both global (quantified in terms of the $W^{s, p}$-norms) and local.

We focus our attention on the $\mathrm{Lip}^{+}$-stability of the viscosity regularization associated with such weakly coupled systems. We derive sufficient conditions, interesting for their own sake, under which the viscosity (and hence the entropy) solutions are $\mathrm{Lip}^{+}$-stable in an appropriate sense. Equipped with this, we may apply the abovementioned convergence rate analysis to approximate solutions that share this type of $\mathrm{Lip}^{+}$-stability.
\end{abstract}

\section{Statement of MAin RESUlts}

We study approximate solutions of weakly coupled nonlinear systems of the form

$$
\frac{\partial}{\partial t} u_{i}(x, t)+\frac{\partial}{\partial x} f_{i}\left(u_{i}(x, t)\right)=\sum_{j} S_{i j} u_{j}(x, t) \quad, \quad 0<t \leq T \quad, \quad i=1, \ldots, n,
$$

subject to compactly supported (or periodic) initial conditions

$$
u_{i}(x, t=0)=u_{i}^{0}(x) \quad, \quad u_{i}^{0} \in L^{\infty} \cap B V \quad, \quad i=1, \ldots, n .
$$

This system takes, formally, the equivalent diagonal form

$$
\frac{\partial}{\partial t} u_{i}+a_{i}\left(u_{i}\right) \frac{\partial}{\partial x} u_{i}=\sum_{j} S_{i j} u_{j} \quad, \quad 0<t \leq T,
$$

where $a_{i}=f_{i}^{\prime}$ (the index $i$ takes henceforth the values $\left.1, \ldots, n\right)$. The system is nonlinear in the sense that each one of its fluxes is convex, i.e., $a_{i}^{\prime} \geq \alpha_{i}>0$.

Received by the editor May 24, 1993

1991 Mathematics Subject Classification. Primary 35L65; Secondary 65M10, 65M15.

This research was supported in part by the Basic Research Foundation, Israel Academy of Sciences and Humanities.

1 Passed away in the dawn of April the 26th, 1994, at age 29, in The Himalayas, Nepal. 
The system (1.1) is weakly coupled in the sense that its coupling is solely due to the low-order terms on the right-hand side. Here, $S=\left\{S_{i j}\right\}$ is a constant matrix whose off-diagonal entries are assumed to be nonnegative,

$$
S_{i j} \geq 0 \quad \forall i \neq j
$$

This nonnegativity assumption yields the $\mathrm{Lip}^{+}$-stability, which is at the heart of our analysis and will be discussed later. The necessity of this assumption in this context is demonstrated in $\S 2$ by a counterexample from [1]. A related maximum principle for such systems satisfying (1.4) may be found in [8].

The statements of existence and uniqueness of entropy solutions in this case may be easily adapted from the scalar " $L^{1}$-type" theory via the viscosity limit and Krushkov entropy condition (Lax, Krushkov [10]). Alternatively, an existence theory for (1.1) may also be derived by the method of fractional steps, in a manner similar to the treatment in [3]. As for uniqueness, our convergence rate results, Theorems 1.4 and 1.6 below, yield uniqueness within the class of $\mathrm{Lip}^{+}$-stable solutions.

Owing to the convexity of the fluxes, $f_{i}=f_{i}\left(u_{i}\right)$, the entropy solutions of (1.1) satisfy a Lip ${ }^{+}$-stability condition, similar to the familiar Oleinik's E-condition from the scalar framework [7], which asserts an a priori upper bound for the Lip $^{+}$ seminorm of the entropy solution. To this end, we let $\|\cdot\|_{\mathrm{Lip}^{+}}$denote the $\mathrm{Lip}^{+}{ }_{-}$ seminorm

$$
\|w(x)\|_{\text {Lip }^{+}}=\operatorname{ess} \sup _{x \neq y}\left(\frac{w(x)-w(y)}{x-y}\right)^{+}, \quad(\cdot)^{+}=\max (\cdot, 0) .
$$

The entropy solutions of (1.1) are those which may be realizable as small viscosity solutions of the parabolic regularization,

$$
\frac{\partial}{\partial t} u_{i}^{\varepsilon}+\frac{\partial}{\partial x} f_{i}\left(u_{i}^{\varepsilon}\right)=\sum_{j} S_{i j} u_{j}^{\varepsilon}+\varepsilon \frac{\partial^{2}}{\partial x^{2}} Q_{i}\left(u_{i}^{\varepsilon}\right), \quad Q_{i}^{\prime}>0, \varepsilon \downarrow 0 .
$$

In $\S 2$ we use this viscosity approximation in order to find a sharp form of the abovementioned a priori bound on the $\mathrm{Lip}^{+}$-seminorm of $a_{i}\left(u_{i}(\cdot, t)\right)$, which we call Lip $^{+}$-stability. We state this as

Theorem 1.1 (Lip $^{+}$-stability estimate). There exists a constant $K$ (depending solely on the constant matrix $S$ and on $\left.\left\|a_{i}^{\prime}\right\|_{L^{\infty}}\right)$, such that $W(t)=$ $\max _{i}\left\|a_{i}\left(u_{i}(\cdot, t)\right)\right\|_{\text {Lip }^{+}}$satisfies

$$
W(t) \leq \frac{e^{K t}}{\frac{1}{W(0)}+\frac{e^{K t}-1}{K}} \leq \max \{W(0), K\}
$$

In this paper we study the convergence rate of approximate solutions to the weakly coupled system (1.1). Let $v_{i}^{\varepsilon}(x, t)$ denote such an approximate solution, which is parameterized with respect to the small parameter $\varepsilon$, e.g., the vanishing viscosity amplitude, a vanishing discretization gridsize, $\varepsilon \sim \Delta x$, or the inverse of an increasing number of modes, $\varepsilon \sim N^{-1}$. We intend to quantify the convergence rate of such approximations in terms of their small scale parameter, $\varepsilon$. This convergence is achieved by establishing $W^{-1,1}$-consistency and Lip ${ }^{+}$-stability, which are appropriately adapted to the current context of nonlinear problems.

In light of Theorem 1.1, we begin with: 
Definition 1.2 (Lip ${ }^{+}$-stability). Let $\left\{v_{i}^{\varepsilon}(x, t)\right\}_{\varepsilon>0}$ be a family of approximate solutions of $(1.1)$ and let $W^{\varepsilon}(t)=\max _{i}\left\|a_{i}\left(v_{i}^{\varepsilon}(\cdot, t)\right)\right\|_{\mathrm{Lip}^{+}}$. This family is called Lip ${ }^{+}$ stable if there exists a constant, $C_{T}$, (independent of $\varepsilon$ ) such that

$$
W^{\varepsilon}(t) \leq C_{T} \quad, \quad 0 \leq t \leq T .
$$

The family is called weakly $\mathrm{Lip}^{+}{ }_{-}$stable if the following weaker conditions hold:

$$
e^{\int_{0}^{T} W^{\varepsilon}(t) d t} \leq \mathcal{O}\left(\frac{1}{\varepsilon}\right) \quad \text { and } \quad \int_{0}^{T} e^{\int_{t}^{T} W^{\varepsilon}(\tau) d \tau} d t \leq \mathcal{O}(|\ln \varepsilon|)
$$

Remark. The above definition stems from Theorem 1.1, where we have to distinguish between two different types of initial data:

- Lip $^{+}$-bounded initial data, $W(0)<\infty$. In this case the entropy solution remains Lip ${ }^{+}$-bounded, (1.7), and we require the same to hold for the approximate solution, [4].

- Lip $^{+}$-unbounded initial data, $W(0)=\infty$. This case indicates the presence of initial rarefactions so that the entropy solution is not $\mathrm{Lip}^{+}$-stable. However, we can smooth out such initial discontinuities (without sacrificing accuracy), to yield mollified rarefaction with $\mathrm{Lip}^{+}$-size of order $W(0) \sim 1 / \varepsilon$. In this case, Theorem 1.1 tells us that the entropy solution satisfies a corresponding weaker form of $\mathrm{Lip}^{+}$stability,

$$
e^{\int_{0}^{T} W(t) d t}=1+W(0) \frac{e^{K T}-1}{K} \leq \mathcal{O}\left(\frac{1}{\varepsilon}\right)
$$

and

$$
\begin{aligned}
\int_{0}^{T} e^{\int_{t}^{T} W(\tau) d \tau} d t & =\frac{1+W(0) \cdot \frac{e^{K T}-1}{K}}{K-W(0)}\left[K T+\ln \left(\frac{1}{1-W(0) \cdot \frac{e^{K T}-1}{K}}\right)\right] \\
& \leq \mathcal{O}(|\ln \varepsilon|) ;
\end{aligned}
$$

this motivates the second part of Definition 1.2 (see also [6]).

For a given family of approximate solutions, $\left\{v^{\varepsilon}\right\}_{\varepsilon>0}$, we let $r^{\varepsilon}$ denote the corresponding local truncation error (residual error),

$$
\frac{\partial}{\partial t} v_{i}^{\varepsilon}+\frac{\partial}{\partial x} f_{i}\left(v_{i}^{\varepsilon}\right)=\sum_{j} S_{i j} v_{j}^{\varepsilon}+r_{i}^{\varepsilon} .
$$

We shall provide error estimates for $e_{i}^{\varepsilon}=v_{i}^{\varepsilon}-u_{i}$ in terms of the size of the truncation error $r_{i}^{\varepsilon}$. The norm which we employ is the $W^{-1,1}\left(\mathbb{R}_{x}\right)$-norm, defined as

$$
\|w(x, t)\|_{W^{-1,1}\left(\mathbb{R}_{x}\right)}=\left\|\int_{-\infty}^{x} w(\xi, t) d \xi\right\|_{L^{1}\left(\mathbb{R}_{x}\right)} .
$$

Note that $\|w\|_{W^{-1,1}}$ is a proper norm only for functions with zero mean, $\int_{\mathbb{R}} w(x, t) d x$ $=0$.

We assume that our approximate solutions are $L^{\infty}$-bounded,

$$
\left\|v_{i}^{\varepsilon}(\cdot, t)\right\|_{L^{\infty}} \leq C_{t}\left\|v_{i}^{\varepsilon}(\cdot, 0)\right\|_{L^{\infty}},
$$

and conservative

$$
\int_{\mathbb{R}} e_{i}^{\varepsilon}(\xi, 0) d \xi=0 \quad, \quad \int_{\mathbb{R}} r_{i}^{\varepsilon}(\xi, t) d \xi=0 .
$$

Differencing (1.10) and (1.1) and integrating with respect to $x$ leads to a system of partial differential equations for $\int^{x} e_{i}^{\varepsilon}(\xi, t) d \xi$. Letting $x \rightarrow \infty$, we obtain, owing to 
conservation, (1.11), a system of homogeneous linear ordinary differential equations for $\int_{\mathbb{R}} e_{i}^{\varepsilon}(\xi, t) d \xi$ with zero initial conditions. This implies that the error has zero mean,

$$
\int_{\mathbb{R}} e_{i}^{\varepsilon}(\xi, t) d \xi=0
$$

Note that the components of the system (1.1) need not be conserved in time, because of the source terms on the right-hand side,

$$
\int_{\mathbb{R}} u(\xi, t) d \xi=e^{S t} \int_{\mathbb{R}} u^{0}(\xi) d \xi
$$

Instead, the total mass of the approximate solutions is required to match the total mass of the exact solution, (1.12); hence, we can measure the error $e_{i}^{\varepsilon}(\cdot, t)$ in terms of the $W^{-1,1}$-norm. Note that (1.12) need not hold in the more general case of nonlinear coupling source terms.

Next, let us define the sense in which these approximate solutions approximate the system, (1.1), and the initial data, (1.2).

Definition 1.3. A family $\left\{v_{i}^{\varepsilon}(x, t)\right\}_{\varepsilon>0}$ of approximations, (1.10), is $W^{-1,1_{-}}$ consistent of order $\delta=\delta(\varepsilon)$ with the initial data, (1.2), if

$$
\left\|e_{i}^{\varepsilon}(\cdot, 0)\right\|_{W^{-1,1}} \leq C \cdot \delta
$$

and with the system, (1.1), if

$$
\left\|r_{i}^{\varepsilon}\right\|_{L^{\infty}\left([0, T], W^{-1,1}\left(\mathbb{R}_{x}\right)\right)} \leq C_{T} \cdot \delta .
$$

Equipped with the above definitions, we turn to the main results of this paper (the proofs of which are given in $\S 3$ ):

Theorem 1.4 (Convergence rate for $\mathrm{Lip}^{+}$-bounded initial data). Consider the system (1.1) subject to the $\mathrm{Lip}^{+}{ }^{-}$bounded initial data, $\max _{i}\left\|u_{i}^{0}\right\|_{\mathrm{Lip}^{+}}<\infty$. Let $\left\{v_{i}^{\varepsilon}(x, t)\right\}_{\varepsilon>0}$ be a family of approximations, (1.10), which is

- $W^{-1,1}$-consistent with the system, (1.1), and the initial data, (1.2), of order $\mathcal{O}(\varepsilon)$

- Lip $^{+}$-stable $(1.8)$.

Then for every $T>0$ there exists a constant $C_{T}$ such that

$$
\left\|e_{i}^{\varepsilon}(\cdot, T)\right\|_{W^{-1,1}} \leq C_{T} \cdot \varepsilon .
$$

Before we turn to the $\mathrm{Lip}^{+}$-unbounded case we define the notion of $L^{1}$-stability.

Definition 1.5. An approximate solution operator, $S^{\varepsilon}(t)$, is $L^{1}$-stable if for any two initial conditions, $u_{0}$ and $v_{0}$,

$$
\left\|S^{\varepsilon}(t) u_{0}-S^{\varepsilon}(t) v_{0}\right\|_{L^{1}\left(\mathbb{R}_{x}\right)} \leq \text { Const }_{t} \cdot\left\|u_{0}-v_{0}\right\|_{L^{1}\left(\mathbb{R}_{x}\right)} \quad, \quad t>0 .
$$

Our second convergence rate result allows possibly $\mathrm{Lip}^{+}$-unbounded initial data.

Theorem 1.6 (Convergence rate for $\mathrm{Lip}^{+}$-unbounded initial data). Consider the system (1.1) subject to the possibly $\mathrm{Lip}^{+}$-unbounded initial data, $\max _{i}\left\|u_{i}^{0}\right\|_{\mathrm{Lip}^{+}}$ $\leq \infty$. Let $\left\{v_{i}^{\varepsilon}(x, t)\right\}_{\varepsilon>0}$ be a family of approximations, (1.10), which is

- $W^{-1,1}$-consistent with the system, (1.1), of order $\mathcal{O}(\varepsilon)$ and with the initial data, (1.2), of order $\mathcal{O}\left(\varepsilon^{2}|\ln \varepsilon|\right)$;

- weakly $\mathrm{Lip}^{+}$-stable (1.9). 
In addition, assume that $v_{i}^{\varepsilon}(\cdot, t)$ are compactly supported for all $0 \leq t \leq T$ and $L^{1}$-stable. Then for every $T>0$ there exists a constant $C_{T}$ such that

$$
\left\|e_{i}^{\varepsilon}(\cdot, T)\right\|_{W^{-1,1}} \leq C_{T} \cdot \varepsilon|\ln \varepsilon| .
$$

Remarks. 1. The $W^{-1,1}$-error estimates of Theorems 1.4 and 1.6 may be translated, along the lines of [4], into the following global $W^{s, p}$-error estimates:

$$
\left\|e_{i}^{\varepsilon}(\cdot, T)\right\|_{W^{s, p}} \leq \mathcal{O}(\tilde{\varepsilon})^{\frac{1-s p}{2 p}} \quad, \quad-1 \leq s \leq \frac{1}{p}, 1 \leq p \leq \infty,
$$

where

$$
\tilde{\varepsilon}= \begin{cases}\varepsilon & \text { if } \max _{i}\left\|u_{i}^{0}\right\|_{\text {Lip }^{+}}<\infty, \\ \varepsilon \ln |\varepsilon| & \text { if } \max _{i}\left\|u_{i}^{0}\right\|_{\text {Lip }^{+}}=\infty\end{cases}
$$

2. These estimates imply also an $\mathcal{O}(\tilde{\varepsilon})^{\frac{1}{3}}$ local error estimate and an $\mathcal{O}(\tilde{\varepsilon})^{\frac{r}{r+2}}$ local error estimate for the postprocessed approximate solution, away from shocks ( $r$ is the degree of local smoothness of the exact solution; for details consult $[6$, $(2.26),(3.9 \mathrm{~b})])$.

The use of $\mathrm{Lip}^{+}$-stability in order to establish convergence rate estimates was first suggested by Tadmor in [11]. It was used in $[4,5,9,12]$ in the context of scalar conservation laws with $\mathrm{Lip}^{+}$-bounded initial data. The case of $\mathrm{Lip}^{+}$-unbounded initial data, i.e., initial rarefactions, was treated in [6], where the idea of using the Lip $^{+}$-stability of $a(u)$ rather than that of $u$ was introduced. In fact, the current paper is an extension of the framework presented in these papers, and especially in [6], for the purpose of dealing with weakly coupled systems.

We note that for scalar conservation laws with no source terms (i.e., $n=1$ and $S=0$ ) we get $K=0$ in (1.7) (see the proof of Theorem 1.1 in $\S 2$ ). Using L'Hospital's rule, we find that

$$
\lim _{K \rightarrow 0} \frac{e^{K t}}{\frac{1}{W(0)}+\frac{e^{K t}-1}{K}}=\frac{1}{\frac{1}{W(0)}+t},
$$

which recovers the familiar strong $\mathrm{Lip}^{+}$-stability from [11]. In a similar manner, we may recover the scalar convergence rate analysis of the abovementioned papers.

We conclude this presentation by noting that the framework suggested in this paper applies, in principle, to the approximate solutions treated in the abovementioned references. In particular, most of the work concerning the viscosity regularization, (1.6), will be done in $\S 2$.

\section{Properties OF The Viscosity AND Entropy SOLUtions}

In this section we study various properties of the smooth viscosity solution, (1.6), which are uniform in $\varepsilon$, in order to conclude similar properties of the entropy solution of the inviscid system (1.1). We discuss most of these properties briefly, as most of the results are straightforward adaptations of the familiar scalar theory.

We start by proving that $\left\{u_{i}^{\varepsilon}\right\}_{\varepsilon>0}$ are uniformly bounded in all $L^{p}$-norms, $1 \leq$ $p \leq \infty$, by the method of $L^{p}$-iterations. For simplicity, we chose $Q_{i}\left(u_{i}\right)=u_{i}$, so that the parabolic regularization (1.6) takes the form

$$
\frac{\partial}{\partial t} u_{i}+\frac{\partial}{\partial x} f_{i}\left(u_{i}\right)=\sum_{j} S_{i j} u_{j}+\varepsilon \frac{\partial^{2}}{\partial x^{2}} u_{i}
$$


(for convenience, we omit the superscript $\varepsilon$ throughout this section). Integrating (2.1) against $p\left|u_{i}^{p-1}\right| \operatorname{sgn}\left(u_{i}\right), 1 \leq p<\infty$, and using the straightforward identities $\left|w^{p}\right|_{y}=p\left|w^{p-1}\right| \operatorname{sgn}(w) w_{y} \quad, \quad\left|w^{p}\right|_{y y}=p\left|w^{p-1}\right| \operatorname{sgn}(w) w_{y y}+p(p-1)\left|w^{p-2}\right| w_{y}^{2}$, and the Hölder inequality,

$$
\int_{x}\left|w^{p-2}\right| w v \leq\|w\|_{L^{p}}^{p-1}\|v\|_{L^{p}}
$$

we arrive at

$$
\frac{d}{d t}\left\|u_{i}\right\|_{L^{p}}^{p} \leq p S_{i i}\left\|u_{i}\right\|_{L^{p}}^{p}+p \sum_{j \neq i} S_{i j} \int_{x}\left|u_{i}^{p-2}\right| u_{i} u_{j} \leq p\left\|u_{i}\right\|_{L^{p}}^{p-1} \sum_{j}\left|S_{i j}\right|\left\|u_{j}\right\|_{L^{p}} .
$$

Assume that $\max _{i}\left\|u_{i}\right\|_{L^{p}}=\left\|u_{m}\right\|_{L^{p}}$. Then (2.2), with $i=m$, implies that

$$
\frac{d}{d t}\left\|u_{m}\right\|_{L^{p}} \leq\left\|u_{m}\right\|_{L^{p}} \sum_{j}\left|S_{m j}\right|
$$

which leads to $L^{p}$-boundedness, $1 \leq p<\infty$,

$$
\max _{i}\left\|u_{i}(\cdot, t)\right\|_{L^{p}} \leq e^{K t} \max _{i}\left\|u_{i}(\cdot, 0)\right\|_{L^{p}} \quad, \quad K=\|S\|_{L^{\infty}}=\max _{i}\left\{\sum_{j}\left|S_{i j}\right|\right\} .
$$

Letting $p \rightarrow \infty$, we get that (2.4) holds for $p=\infty$ as well.

Remark. General viscosity coefficients, $Q_{i}\left(u_{i}\right), Q_{i}^{\prime}>0$, may be treated in a similar manner, using the inequality

$$
\varepsilon p \int_{x}\left|w^{p-1}\right| \operatorname{sgn}(w) \frac{\partial^{2}}{\partial x^{2}} Q_{i}\left(u_{i}\right)=-\varepsilon p(p-1) \int_{x} Q_{i}^{\prime}\left(u_{i}\right)\left|w^{p-2}\right| w_{x}^{2} d x \leq 0 .
$$

Next, we refer to $L^{1}$-stability in the sense of Definition 1.5. The idea is similar to the proof of $L^{1}$-contraction, proposed in [2], for scalar conservation laws; the only difference in our case is due to the coupling, which gives a (stable) growth in time, governed by the same constants as in the previous proof, i.e,

$$
\left\|v_{i}(\cdot, t)-u_{i}(\cdot, t)\right\|_{L^{1}} \leq e^{K t}\left\|v_{i}(\cdot, 0)-u_{i}(\cdot, 0)\right\|_{L^{1}},
$$

where $u_{i}$ and $v_{i}$ are any two viscosity solutions of (1.6). This implies uniqueness for the viscosity solutions as well as for their limit, the entropy solutions.

The $B V$-boundedness of the solutions,

$$
\left\|u_{i}(\cdot, t)\right\|_{B V} \leq e^{K t}\left\|u_{i}(\cdot, 0)\right\|_{B V},
$$

may be derived from (2.5) using the translation invariance of (1.6). The BVboundedness of the viscosity solution is used in order to prove its $W^{-1,1}$-consistency with entropy solution, see $\S 3$.

We now turn to the essential property of $\mathrm{Lip}^{+}$-stability.

Proof of Theorem 1.1. Let us consider the parabolic regularization of (1.1) which corresponds to the choice $Q_{i}=a_{i}$,

$$
\frac{\partial}{\partial t} u_{i}+\frac{\partial}{\partial x} f_{i}\left(u_{i}\right)=\sum_{j} S_{i j} u_{j}+\varepsilon \frac{\partial^{2}}{\partial x^{2}} a_{i}\left(u_{i}\right) .
$$


Multiplying (2.7) by the diagonal matrix $\operatorname{diag}\left\{a_{i}^{\prime}\right\}$, we get that

$$
\frac{\partial}{\partial t} a_{i}+a_{i} \frac{\partial}{\partial x} a_{i}=a_{i}^{\prime} \sum_{j} S_{i j} u_{j}+\varepsilon a_{i}^{\prime} \frac{\partial^{2}}{\partial x^{2}} a_{i} .
$$

Differentiating (2.8) with respect to $x$ and rearranging, we find that $w_{i}=\frac{\partial}{\partial x} a_{i}$ satisfies

$$
\frac{\partial}{\partial t} w_{i}+w_{i}^{2}+A_{i} \frac{\partial}{\partial x} w_{i}=\sum_{j} C_{i j} w_{j}+\varepsilon a_{i}^{\prime} \frac{\partial^{2}}{\partial x^{2}} w_{i}
$$

here,

$$
A_{i}=a_{i}-\varepsilon \frac{a_{i}^{\prime \prime}}{a_{i}^{\prime}} w_{i} \quad, \quad C_{i i}=S_{i i}+\frac{a_{i}^{\prime \prime}}{a_{i}^{\prime}} \sum_{j} S_{i j} u_{j} \quad, \quad C_{i j}=S_{i j} \frac{a_{i}^{\prime}}{a_{j}^{\prime}}, \quad i \neq j .
$$

We concentrate now on the maximal values of $w_{i}$ which may be attained at different points, say $x_{i}(t)$,

$$
W_{i}(t)=\max _{x}\left\{w_{i}(x, t)^{+}\right\}=w_{i}\left(x_{i}(t), t\right) \geq 0 .
$$

At these points we have

$$
\frac{\partial}{\partial x} w_{i}\left(x_{i}(t), t\right)=0 \quad, \quad \frac{\partial^{2}}{\partial x^{2}} w_{i}\left(x_{i}(t), t\right) \leq 0 .
$$

Using this in (2.9), we arrive at the coupled system of ordinary differential inequalities

$$
\frac{d}{d t} W_{i} \leq-W_{i}^{2}+\sum_{j} C_{i j} W_{j}
$$

Here we made use of the nonnegativity assumption (1.4), which-together with the positivity of $a_{i}^{\prime}$-guarantees that $C_{i j} \geq 0$ whenever $i \neq j$. This implies that

$$
C_{i j} w_{j}\left(x_{i}, t\right) \leq C_{i j} w_{j}\left(x_{j}, t\right)=C_{i j} W_{j}(t) \quad \forall i \neq j
$$

and (2.11) is therefore justified.

We note that the coefficients $C_{i j}$ are bounded, owing to the $L^{\infty}$-bound established earlier for $u_{i}$ and the assumption of convexity, $a_{i}^{\prime} \geq \alpha_{i}>0$.

From (1.4) and (2.11) we conclude that $W=\max _{i} W_{i}$ satisfies the Riccati-type inequality

$$
\frac{d}{d t} W \leq-W^{2}+K W \quad, \quad K=\max _{i}\left\{\sum_{j} C_{i j}\right\}
$$

Multiplying (2.12) by $e^{-K t}$, we get that $Z=e^{-K t} W$ is governed by

$$
\frac{d}{d t} Z \leq-e^{K t} Z^{2}
$$

Finally, integration of (2.13) yields, for $W=e^{K t} Z$, the desired estimate (1.7). 
Remarks. 1. General viscosity coefficients $Q_{i} \neq a_{i}$, may be treated at the expense of third-order perturbations, along the lines of [6, Lemma 3.1]. The perturbated Riccati inequality will take the form

$$
\frac{d}{d t} W_{i} \leq-W_{i}^{2}+\sum_{j} C_{i j} W_{j}+\varepsilon B_{i} W_{i}^{3}, \quad B_{i}=\frac{1}{a_{i}^{\prime}}\left(\frac{Q_{i}^{\prime}}{a_{i}^{\prime}}\right)^{\prime \prime} .
$$

2. The $\mathrm{Lip}^{+}$-stability proof, similar to the one presented here, could be applied to the more general case of nonlinear coupling. Let us look at a weakly coupled viscosity system

$$
\frac{\partial}{\partial t} u_{i}+\frac{\partial}{\partial x} f_{i}\left(u_{i}\right)=S_{i}\left(u_{1}, \ldots, u_{n}\right)+\varepsilon \frac{\partial^{2}}{\partial x^{2}} a_{i}\left(u_{i}\right),
$$

and assume that the coupling source functions, $S_{i}$, satisfy

$$
0 \leq \frac{\partial S_{i}}{\partial u_{j}} \leq C \quad \forall i \neq j \quad \text { and } \quad\left|\frac{\partial S_{i}}{\partial u_{i}}\right| \leq C
$$

Then the viscosity solution of (2.15) and, consequently, the limit entropy solution are $\mathrm{Lip}^{+}$-stable.

Example. Before we conclude this section, we would like to emphasize the necessity of the nonnegativity assumption (1.4). This assumption was used, mainly, for the derivation of inequality (2.11). Observe that if (1.4) fails, a shock wave of, say, $u_{j}$, may give positive contribution to $w_{i}$ in (2.9), and a rarefaction wave might evolve, violating the $\mathrm{Lip}^{+}$-stability.

This situation occurs in the $2 \times 2$ systems presented in [1] and [3]. We will briefly discuss the system presented in $[1, \S 3]$, where there are two coupled Burgers' equations of the form

$$
\begin{aligned}
& u_{t}+u u_{x}+v=0, \\
& v_{t}+v v_{x}=0 .
\end{aligned}
$$

The linear coupling matrix in this case, $S=\left(\begin{array}{cc}0 & -1 \\ 1 & 0\end{array}\right)$, violates (1.4).

In [1], Hunter presents numerical solutions of two examples; one with the initial data

$$
\begin{aligned}
& u(x, 0)=\sin (x), \\
& v(x, 0)=\cos (x),
\end{aligned}
$$

$[1,(3.6)]$, and the other with the initial data

$$
\begin{aligned}
& u(x, 0)=0, \\
& v(x, 0)=s(x-\pi),
\end{aligned}
$$

where $s(x)$ is the $2 \pi$-periodic sawtooth function,

$$
s(x)=x,|x|<\pi \quad \text { and } \quad s(x+2 \pi)=s(x),
$$

$[1,(3.9)]$. The numerical results for these two examples are given in $[1$, Figures 1 and 2]. In both examples, rarefactions evolve in a finite time and there is no $\mathrm{Lip}^{+}$-stability. Moreover, it is clearly observed, in both numerical solutions, that the rarefaction in one variable occurs exactly in the same space location as the shock in the other variable, as explained by our above analysis. 
This situation is prevented in our case by condition (1.4), which guarantees $\mathrm{Lip}^{+}$-stability, meaning that a rarefaction wave cannot develop and that initial rarefactions immediately open.

\section{Proof of the main Results}

Here we prove our convergence rate estimates stated in Theorems 1.4 and 1.6. To this end, we introduce the following notation, which links together the Lip ${ }^{+}$-stability of both the approximate solution, $v_{i}^{\varepsilon}(x, t)$, and the entropy solution, $u_{i}(x, t)$ :

$$
L(t)=\max _{i} L_{i}(t) \quad, \quad L_{i}(t)=\max \left\{\left\|a\left(v_{i}^{\varepsilon}(\cdot, t)\right)\right\|_{\mathrm{Lip}^{+}},\left\|a\left(u_{i}(\cdot, t)\right)\right\|_{\mathrm{Lip}^{+}}\right\} .
$$

Proof of Theorem 1.4. Assume that both the exact and the approximate initial data are $\mathrm{Lip}^{+}$-bounded,

$$
L(0)<\infty .
$$

Subtracting (1.1) from (1.10), we arrive at the equation which governs the error, $e_{i}^{\varepsilon}(x, t)$,

$$
\frac{\partial}{\partial t} e_{i}^{\varepsilon}+\frac{\partial}{\partial x}\left[\bar{a}_{i} e_{i}^{\varepsilon}\right]=\sum_{j} S_{i j} e_{j}^{\varepsilon}+r_{i}^{\varepsilon}
$$

where

$$
\bar{a}_{i}=\bar{a}_{i}\left(v_{i}^{\varepsilon}, u_{i}\right)=\int_{0}^{1} a_{i}\left(\xi v_{i}^{\varepsilon}+(1-\xi) u_{i}\right) d \xi .
$$

Integration of (3.2) with respect to $x$ gives

$$
\frac{\partial}{\partial t} E_{i}^{\varepsilon}+\bar{a}_{i} \frac{\partial}{\partial x} E_{i}^{\varepsilon}=\sum_{j} S_{i j} E_{j}^{\varepsilon}+R_{i}^{\varepsilon}
$$

where $E_{i}^{\varepsilon}$ and $R_{i}^{\varepsilon}$ denote the primitives of $e_{i}^{\varepsilon}$ and $r_{i}^{\varepsilon}$, respectively,

$$
E_{i}^{\varepsilon}(x, t)=\int_{-\infty}^{x} e_{i}^{\varepsilon}(\xi, t) d \xi \quad, \quad R_{i}^{\varepsilon}(x, t)=\int_{-\infty}^{x} r_{i}^{\varepsilon}(\xi, t) d \xi .
$$

Note that, owing to conservation, (1.12), we get that

$$
\lim _{x \rightarrow \infty} E_{i}^{\varepsilon}(x, t)=\int_{-\infty}^{\infty} e_{i}^{\varepsilon}(\xi, t) d \xi=0 .
$$

Integration of (3.4) against $\operatorname{sgn}\left(E_{i}^{\varepsilon}\right)$ yields

$$
\frac{d}{d t}\left\|E_{i}^{\varepsilon}\right\|_{L^{1}} \leq-\int_{x} \bar{a}_{i} \frac{\partial}{\partial x}\left|E_{i}^{\varepsilon}\right| d x+\sum_{j} S_{i j}\left\|E_{j}^{\varepsilon}\right\|_{L^{1}}+\left\|R_{i}^{\varepsilon}\right\|_{L^{1}} .
$$

To proceed, we use the following result, taken from [6] (consult (2.11) and (2.13) there),

$$
\int_{x} \bar{a}_{i}\left(-\frac{\partial}{\partial x}\left|E_{i}^{\varepsilon}\right|\right) d x \leq L_{i}(t) \cdot\left\|E_{i}^{\varepsilon}\right\|_{L^{1}}
$$

Combining (3.6) and (3.7), we get

$$
\frac{d}{d t}\left\|E_{i}^{\varepsilon}\right\|_{L^{1}} \leq L_{i}(t)\left\|E_{i}^{\varepsilon}\right\|_{L^{1}}+\sum_{j} S_{i j}\left\|E_{j}^{\varepsilon}\right\|_{L^{1}}+\left\|R_{i}^{\varepsilon}\right\|_{L^{1}} .
$$


Introducing the notations

$$
E^{\varepsilon}(t)=\max _{i}\left\{\left\|E_{i}^{\varepsilon}(\cdot, t)\right\|_{L^{1}}\right\} \quad \text { and } \quad R^{\varepsilon}=\max _{0 \leq t \leq T} \max _{i}\left\{\left\|R_{i}^{\varepsilon}(\cdot, t)\right\|_{L^{1}}\right\},
$$

inequality (3.8) implies that

$$
\frac{d}{d t} E^{\varepsilon}(t) \leq[L(t)+K] E^{\varepsilon}(t)+R^{\varepsilon} \quad, \quad K=\max _{i}\left\{\sum_{j} S_{i j}\right\} .
$$

The solution of the above differential inequality gives

$$
E^{\varepsilon}(T) \leq e^{K T+\int_{0}^{T} L(t) d t}\left[E^{\varepsilon}(0)+R^{\varepsilon} \int_{0}^{T} e^{-K s-\int_{0}^{s} L(\tau) d \tau} d s\right] .
$$

This inequality emphasizes the main ingredients of our error estimate:

- The Lip ${ }^{+}$-stability - measured by the temporal integrals of $L(t)$

- The $W^{-1,1}$-consistency with the system and the initial data-measured, respectively by $R^{\varepsilon}$ and $E^{\varepsilon}(0)$.

The Lip ${ }^{+}$-stability of the entropy solution, (1.7), together with that of the approximate solution, (1.8), and assumption (3.1), imply that

$$
L(t) \leq C_{T} \quad, \quad 0 \leq t \leq T .
$$

The uniform Lip $^{+}$-bound, (3.10), and inequality (3.9) imply that

$$
E^{\varepsilon}(T) \leq e^{\left(K+C_{T}\right) T} E^{\varepsilon}(0)+\frac{e^{\left(K+C_{T}\right) T}-1}{K+C_{T}} R^{\varepsilon} .
$$

Since we assumed $W^{-1,1}$-consistency of order $\mathcal{O}(\varepsilon)$ with the initial condition and with the system, both $E^{\varepsilon}(0)$ and $R^{\varepsilon}$ are of order $\mathcal{O}(\varepsilon)$ and, therefore,

$$
E^{\varepsilon}(T) \leq \mathcal{O}(\varepsilon) .
$$

Hence, we conclude, componentwise, that

$$
\left\|e_{i}^{\varepsilon}(T)\right\|_{W^{-1,1}}=\left\|E_{i}^{\varepsilon}(T)\right\|_{L^{1}} \leq \mathcal{O}(\varepsilon)
$$

and the proof is thus completed.

Proof of Theorem 1.6. Because of the possible existence of initial rarefactions, condition (3.1) need not hold now. Therefore, we introduce the function $\psi_{\delta}(\cdot)=$ $\frac{1}{\delta} \psi(\dot{\delta}), \delta>0$, which is the dilated mollifier of

$$
\psi(x)= \begin{cases}1, & |x| \leq 1 \\ 0, & |x|>1\end{cases}
$$

This choice of mollifier satisfies

$$
\left\|\psi_{\delta} * w-w\right\|_{L^{1}} \leq \mathcal{O}(\delta) \cdot\|w\|_{B V} \quad \text { and } \quad\left\|\psi_{\delta} * w\right\|_{\text {Lip }^{+}} \leq \mathcal{O}\left(\frac{1}{\delta}\right), \quad \delta \downarrow 0 .
$$

With this in mind, we return to the exact system, (1.1), and the approximate one, (1.10), and define a new pair of solutions, $u_{i}^{\delta}$ and $v_{i}^{\varepsilon, \delta}$, by mollifying the initial data; i.e., $u_{i}^{\delta}$ is the solution of (1.1) with the initial data $u_{i}^{\delta}(\cdot, 0)=\psi_{\delta} * u_{i}(\cdot, 0)$ and $v_{i}^{\varepsilon, \delta}$ is the solution of (1.10) with the initial data $v_{i}^{\varepsilon, \delta}(\cdot, 0)=\psi_{\delta} * v_{i}^{\varepsilon}(\cdot, 0)$. 
We are now able to estimate the $W^{-1,1}$-error by decomposing it as follows:

$$
\begin{aligned}
\left\|e_{i}^{\varepsilon}(\cdot, T)\right\|_{W^{-1,1}} \leq & \left\|v_{i}^{\varepsilon}(\cdot, T)-v_{i}^{\varepsilon, \delta}(\cdot, T)\right\|_{W^{-1,1}}+\left\|v_{i}^{\varepsilon, \delta}(\cdot, T)-u_{i}^{\delta}(\cdot, T)\right\|_{W^{-1,1}} \\
& +\left\|u_{i}^{\delta}(\cdot, T)-u_{i}(\cdot, T)\right\|_{W^{-1,1}}
\end{aligned}
$$

Since, for compactly supported functions $w$, it holds that

$$
\|w\|_{W^{-1,1}} \leq|\operatorname{supp}\{w\}| \cdot\|w\|_{L^{1}}
$$

and since both the exact and approximate solutions are $L^{1}$-stable, we bound the first and last terms on the right-hand side of (3.11) as follows $(\Omega$ denotes the common compact support $)^{2}$ :

$$
\begin{aligned}
\| v_{i}^{\varepsilon}(\cdot, T) & -v_{i}^{\varepsilon, \delta}(\cdot, T)\left\|_{W^{-1,1}} \leq|\Omega| \cdot\right\| v_{i}^{\varepsilon}(\cdot, T)-v_{i}^{\varepsilon, \delta}(\cdot, T) \|_{L^{1}} \\
& \leq|\Omega| \cdot C_{T} \cdot\left\|v_{i}^{\varepsilon}(\cdot, 0)-v_{i}^{\varepsilon, \delta}(\cdot, 0)\right\|_{L^{1}} \leq|\Omega| \cdot \mathcal{O}(\delta) \cdot\left\|v_{i}^{\varepsilon}(\cdot, 0)\right\|_{B V} \\
& =\mathcal{O}(\delta) \cdot\left\|v_{i}^{\varepsilon}(\cdot, 0)\right\|_{B V} .
\end{aligned}
$$

Similarly,

$$
\left\|u_{i}(\cdot, T)-u_{i}^{\delta}(\cdot, T)\right\|_{W^{-1,1}} \leq \mathcal{O}(\delta) \cdot\left\|u_{i}(\cdot, 0)\right\|_{B V} .
$$

We are left with the term $\left\|v_{i}^{\varepsilon, \delta}(\cdot, T)-u_{i}^{\delta}(\cdot, T)\right\|_{W^{-1,1}}$. Since

$$
L_{i}^{\delta}(0)=\max \left\{\left\|a_{i}\left(v_{i}^{\varepsilon, \delta}(\cdot, 0)\right)\right\|_{\mathrm{Lip}^{+}},\left\|a_{i}\left(u_{i}^{\delta}(\cdot, 0)\right)\right\|_{\mathrm{Lip}^{+}}\right\} \leq \mathcal{O}\left(\frac{1}{\delta}\right),
$$

condition (3.1) is met by these two initial conditions. Therefore, we conclude, along the lines of the proof of Theorem 1.4 and using the Lip ${ }^{+}$-stability of the entropy and approximate solutions, (1.7) and (1.9), that

$$
\begin{aligned}
E^{\varepsilon, \delta}(T) & \leq e^{K T+\int_{0}^{T} L(t) d t}\left[E^{\varepsilon, \delta}(0)+R^{\varepsilon} \int_{0}^{T} e^{-K s-\int_{0}^{s} L(\tau) d \tau} d s\right] \\
& \leq \mathcal{O}\left(\frac{1}{\delta}\right) E^{\varepsilon}(0)+\mathcal{O}(|\ln \delta|) R^{\varepsilon} .
\end{aligned}
$$

Hence, using (3.11), (3.13) and (3.14) in this order, we get

$$
E^{\varepsilon}(T) \leq \mathcal{O}(\delta)\left[\sum_{i}\left[\left\|u_{i}(\cdot, 0)\right\|_{B V}+\left\|v_{i}^{\varepsilon}(\cdot, 0)\right\|_{B V}\right]\right]+\mathcal{O}\left(\frac{1}{\delta}\right) E^{\varepsilon}(0)+\mathcal{O}(|\ln \delta|) R^{\varepsilon}
$$

Taking $\delta=\mathcal{O}(\varepsilon)$ and using the assumed $W^{-1,1}$-consistency of the approximate solution with both the system and the initial data we have, for each of the error components,

$$
\left\|e_{i}^{\varepsilon}\right\|_{W^{-1,1}}=\left\|E_{i}^{\varepsilon}(t)\right\|_{L^{1}} \leq\left\|E^{\varepsilon}(t)\right\|_{L^{1}} \leq \mathcal{O}(\varepsilon|\ln \varepsilon|),
$$

and the proof is thus concluded.

${ }^{2}$ Note that for viscosity-like approximations, which are not compactly supported, we may use the exponential decay of the tail. 


\section{REFERENCES}

1. J.K. Hunter, "Interacting weakly nonlinear hyperbolic and dispersive waves", in Microlocal Analysis and Nonlinear Waves, M. Beals, R.B. Melrose and J. Rauch, Editors, Springer-Verlag, New York (1991). MR 92e:35103

2. P.D. Lax, "Hyperbolic Systems of conservation laws and the mathematical theory of shock waves", in Regional Conf. Series Lectures in Applied Math. Vol. 11 (SIAM, Philadelphia, 1972). MR 50:2709

3. A. Majda, R.R. Rosales and M. Schonbek, "A canonical system of integro-differential equations arising in resonant nonlinear acoustics", Stud. Appl. Math. Vol. 79 (1988), pp. 205-262. MR 90g:76077

4. H. Nessyahu and E. Tadmor, "The convergence rate of approximate solutions for nonlinear conservation laws", Siam J. on Numer. Anal., Vol. 29 (1992), pp. 1505-1519. MR 93j:65139

5. H. Nessyahu, E. Tadmor and T. Tassa, "The convergence rate of Godunov type schemes", Siam J. on Numer. Anal., Vol. 31 (1994), pp. 1-16. MR 94m:65140

6. H. Nessyahu and T. Tassa, "Convergence rate of approximate solutions to conservation laws with initial rarefactions", Siam J. on Numer. Anal., Vol. 31 (1994), pp. 628-654. CMP 94:12

7. O. A. Oleinik, "Discontinuous solutions of nonlinear differential equations ", Amer. Math. Soc. Transl. (2), Vol. 26 (1963), pp. 95-172. MR 20:1055; MR 27:1721

8. Protter and Weinberger, "Maximum principles in Differential Equations", Prentice-Hall, Englewood Cliffs, NJ, (1967). MR 36:2935

9. S. Schochet and E. Tadmor, "The regularized Chapman-Enskog expansion for scalar conservation laws", Arch. Rational Mech. Anal., Vol. 119 (1992), pp. 95-107. MR 93f:35191

10. J. Smoller, "Shock Waves and Reaction-Diffusion Equations", Springer-Verlag, New York (1983). MR 84d:35002

11. E. Tadmor, "Local error estimates for discontinuous solutions of nonlinear hyperbolic equations", Siam J. on Numer. Anal., Vol. 28 (1991), pp. 891-906. MR 92d:35190

12. E. Tadmor, "Total variation and error estimates for spectral viscosity approximations", Math. of Comp., Vol. 60 (1993), pp. 245-256. MR 93d:35098

School of Mathematical Sciences, Tel-Aviv University, Tel-Aviv 69978, Israel 